\title{
Transzendenz und Immanenz des Bodhicittam
}

\section{_ Eine Methodologie _ Kumataro Kawada}

\section{Problemstellung}

Hier wird das bodhicittam als ein Terminus Technicus der buddhistischen Philosophie gebraucht. Insofern ist es eben das anuttara-samyak-sambodhicittam, m. a. W. abhisambodhicittam. Denn, es bedeutet den Geist (cittam), der die oberste, richige und allgemeine Wahrheit zu er-wachen (abhi-sam- $\sqrt{\text { budh }) ~ s u c h t . ~(D a s ~ c i t t a m ~ h a t ~ k e i n ~ d e u t s c h e s ~ W o r t, ~ d a s ~ s e i n e ~}$ Bedeutung adäquat übertragen kann, weil es dynamisch-kinetische und gesetzmässige Sammlung der Handlungen des Denkens, des Sagens und des körperlichen Benehmens bedeutet, kurz, eine Sammlung der Handlungen, d. h. karmāṇi. Wegen der leichteren Zugangs wird hier 《der Geist》 gebraucht.) Man erhebt anlässlich des Vergänglichkeit aller Dinge das bodhicittam von sich selbt (bodhicittotpāda), was aus lauter freiem Willen getan wird, weil der pratītyasamutpāda, der sogenannte Kausalnexus, der Kausalnexus der Handlungen ist und jeder in diesem Augenblick sich entschliessen kann, entweder das oberste Wissen zu suchen oder in der Unwissenheit schlafen zu bleiben. Und die Erreichung oder das Er-wachen des obersten Wissens ist möglich durch die Transzendenz, das Hinaus-und Übergehen des samisāra, des Rades von Geburt und Tod, des Traidhātuka. Darum ist die Transzendenz des bodhicittam so ausführlich und genau anlysiert und bestimmt, sowohl in Hīnayāna als auch in Mahāyāna, dass es scheint, als ob was wichtig sei, das nur die Transzendenz sei. Aber in der Tat, was wichtig ist, das sind die Transzendenz und die Immanenz des bodhicittam, weil das apratisthitanirvāṇa (das Erlöschen mit keinem Halt) der höchste Stand ist. Das bodhicittam soll nicht nur aus dem sam- 
sāra transzendieren und das oberste Wissen erwachen, auch soll es mit dem Wissen wieder in den sam̄āara zurückkommen und darin das Erlösungswerk fortsetzen. Nach der traditionellen buddhistischen Philosophie ist das Obige der Kernpunkt dessen, was Transzendenz und Immanenz des bodhicittam anbetrifft. Aber dieses Denken bleibt innerhalb der buddhistischen Philosophie stehen, d. h. es ist lauter immanent dem Buddhismus. Wenn man seine Philosophe auf der Ebene der Universalgeschichte der Philosophie betrachtet, ist es selbstverständhich, dass sie nicht die einzige Philosophie ist. Sie steht neben Anderen in jedem Lande und in jeder Zeit. Insofern ist sie eine unter vielen. Diesem universal philosophiegeschichtlichen Tatbestand entsteht ein anderes und neues Problem in Bezug auf Transzendenz und Immanenz des bodhicittam : wie verhält es sich im Angesicht dieses Tatbestandes, um sein Wahrheitsgehalt klar zu machen? Um dieses Problem zu lösen, wären viele Wege, deren einen hier gegangen wird: das bodhicittam in Verhältnis zu der obersten Wahrheit (dharmatā).

\section{Bodhicittam und Dharmatā}

(1) Der Meister

AN, catukkanipāto, 1.Teil, 1. Kapitel (bhaṇḍagāmavaggo) erwähnt die vier Wahrheiten (dhamma): sīla(Sittlichkeit), samādhi(Meditation), paññā (vornehmer Intellect, d. h. der durch sīla und samādhi begründete Intellect) und vimutti anuttarā (die oberste Erlösung). Sie sind die zwei Wahrheiten, nämlich die des zum Erlöschen des Scmerzes führenden Weges und die der Erlöschung des Schmerzes, weil die ersteren drei nichts anderes als der achtgliedrige Weg sind. Im dritten Kapitel (uruvelavaggo) wird erzählt vom Entschluss des Gautama, dass er die vierfältige Wahrheit, die er erwacht hat, als seinen Meister verehren und unter ihrem beständigen Einfluss leben wolle. Der Gedanke, dass die Wahrheit der Meister ist, ist ererbt und fortgesetzt durch pañcavimínatisāhasrikā prajñāpāramitā und das Mahāyānaparinirvāṇa sūtram (dessen vollstädiger Sanskrit-Text noch nicht und nur karge Fragmente gefunden sind). Aus diesen Stellen kommt heraus, dass der Gedanke ein Grundsatz ist, der das Verhältnis des bodhittam zu der erwach- 
Transzendenz und Immanenz des Bodhicittam (Kumataro Kawada) (23)

ten Wahrheit also bestimmt. Die zu erwachende Wahrheit (dharmatā) ist von dem buddha, bodhisattva und so von dem erhoben bodhicittam unabhäng ig ; und die letzteren sind von ihr abhängig. Der junge Prinz Siddhārtha hegteeinen heiligen Wunsch, d. h. erhob sich das bodhicittam und besuchte zwei Meister nach dem Andern. Aber ihre Lehren konnten ihn nicht zufrieden machen. Und so erwachte er, erwarb er die heilige vierfältige Wahrheit mit eigener Anstrengung, ohne einen Meister. Also ist die Wahrheit des buddha (buddhadharma) das Wissen ohne Meister. Aber sie wurde weder von ihm erdichtet noch von ihm willkürlich gesetzt. Daher 《sind diese Wahrheiten vom angesehenen Gautama so erwacht wie sie sind. (1) Die Wahrheit ist die des cittam, was die Besonderheit der Wiss-Sicht des buddha (tathāgata-jñāna-darśanam) ist. Diese Wahrheit, die solche Besonderheit hat, wird von verschiedenen und wichtigen Sutren betont. Z. B. 1.) SN, Nidānasamyttta, Sutta 20 , betont dasselbe in Bezugauf auf den sog. Kausalnexus (pațiccasamuppādo), welcher dasselbe mit der heiligen vierfältigen Wahrheit ist. 2.) Das Daśabhūmikam setzt diesen Gedanken fort. 《Ungeachtet der Ersheinung der tathāgatās (pl.) in die Welt oder der Nicht-Erscheinung bleibt die Wahrheit stehen; diese Beständigkeit der Wahrheit ändert sich nicht. Diese bestehende Wahrheit besteht in der Leere aller Dinge und in der Unwissbarkeit aller Dinge. Nicht nur erscheinen die tathāgatās in die Welt auf Grund dieser Wahrheit, sondern auch erreichen alle śrāvakās und pratyekabuddhās die Wahrheit so wie sie ist.) Die Unwissbarkeit (anupalabdhi) in der Anführung bedeutet nicht den Agnostizismus, sondern Erfassung der Wahrheit mit der prajñā, die eigentlich jenseits des Gegensatze des Subjekts und des Objekts wirkt, und deren Wissen nicht als das im gewöhnlichen Sinne genommen werden

(1) SRT, Bd. XXI, S. 2.

(2) SRT, Bd. XVI, S. 30.

(3) Daśabhümikam, hrsgbn. von J. Rahder, S. 65. Vgl. Daśabhūmīśvara, hrsgbn. von R. Kondo,S. 136.

(4) Taisho Tripițakam, hrsgbn. von J. Takakusu und Anderen, Bd. XII, SS. 387 C; 627 C. 
kann. 3.) Das Mahāyānaparinirvāṇa sūtram lehrt, dass der tathāgata permanent ist und alle Lebwesen die Buddhaheit haben. Und zum Beweis des ersteren Satzes sagt das Sütra, dass der Meister der buddhās die Wahrheit ist, und weil die Wahrheit permanent ist, so die buddhās permanent sind. 4.) Das Lañkāvatāra Sūtra setzt diesen Gedanken fort. 《Ich sagte das, indem ich zwei Wahrheiten im Sinne hatte. Welche zwei ? Sowohl die erwachte Wahrheit (pratyātmadharmatā) als auch die von Alters her bestehende Wahrheit (paurānasthitidharmatā). $\left.)^{(5)}\right\rangle$ Die leteztere ist der dharma als Meister im eigentlichen Sinne und die erstere bedeutet ihre Verehrung und das von ihr beständig beeinflusst Werden.

(2) Das Erwachen der bestehenden Wahrheit

Ihre Verehrung und Einfluss besteht darin, dass man sie in sich verwirklicht, dass man sie ohne einen persönlichen Meister mit eigener Kraft erwacht und dass man nach ihr denkt, sagt und körperlich sich benimmt. Dieses Geschehen wird im siebenten Kapitel (pūrvayoga) des Saddharmapṇd̦arīka erzählt in der Form eines Mythos, welcher Gautama Buddha als Kern hat. Nach dem Mythos erreichte Mahā-abhijñā-jn̄āna-abhibhūr Buddha, die Wahrheit, nachdem er auf dem Platz der bodhi durch zehn kleine kalpās hindurch meditierte. Dieser Mythos zeigt, wie es schwer ist, aus der bestehenden Wahrheit die erwachte Wahrheit zu machen, und dass die bestehende Wahrheit anfanglos ist. Nun, was war die erwachte oder die in sich verwirklichte Wahrheit? Es war, wie das Kapitel deutlich zeigt, die heilige vierfältige Wahrheit und die Solchheit (tathatā) des pratītyasamutpāda. Die beiden Wahrheiten sind ein und dasselbe. Und diese eine Wahrheit ist das eine Fahrzeug (ekayāna) des Saddharmapuṇụarīka, das in die Urquelle des Wahrheit (dharmadhatu) führt, an der śravakās, pratyekabuddhās und bodhisattvās sich je auf 'eigene Weise teilnehmen, die also der ihnen gemeinsame Entstehungsgrund ist. Es ist die Wahrheit, die das Avatamsakam also ausdrückt: 《Geist (cittam), Buddha und Lebwesen (sattva) sind ein und dasselbe, zwischen denen kein Unterschied $z u$ bemerken ist. $\left.{ }^{(7)}\right\rangle$ Und

(5) The Lankavatara Sutra, hrsgbn. vion Bunyiu Nanjio, S. 143.

(6) Saddharmapuṇụarika, hrsgbn. von H. Kern und B. Nanjio, S. 181. 
Transzendenz und Immanenz des Bodhicittam (Kumataro Kawada) (25)

auch 《der Geist (cittam) bringt alles hervor, es gibt nichts, was vom Geist nicht hervorgebracht wird. $)$ Es ist die cittadharmatā (die Grundwahrheit des Geites).

(3) Absolutheitsanspruch

Diese Wahrheit hat Absolutheitsanspruch. Nach dem fünfzehnten Kapitel, betielt 《Lebensdauer des tathāgata》, des Saddharmapuṇdarīka sagt Gautama Buddha: 《damals kam ich zum Bewusstsein der obersten Erkenntnis (agra-bodhi).) Diese oberste Erkenntnis drückt den Absolutheitsanspruch der Wiss-Sicht des buddha am treffendsten aus, weil alle anderen Erkenntnisse und Lehren aus ihr entspringen sollen. Aber hierin steckt eine wichtige und schwierige Frage, sowohl innerhalb der buddhistischen Philosophie als auch in Bezug auf die Universalgeschichte der Philosophie. Der geschichtlich gegebene Buddhismus, sowohl exoterischer als auch esoterischer, bejaht diesen Anspruch, je auf eigene Weise. Der exoterischer Buddhismus besteht auf die Absolutheit der pratyātmadharmatā, die gegründet ist auf die paurāṇasthitidharmatā oder den dharmadhātu. Dagegen macht der esoterische Buddhismus aus der paurāṇasthitidharmatā das dharmadhatvātmakam jñānam und der. Buddha, der mit diesem jñānam begabt ist, wird als Ursprung aller buddhās betrachtet. Und so besteht ein Zwist zwischen beiden Richtungen, der daher kommt, dass der eine die paurāna: sthitidharmatā nicht als Ursprungsbuddha behandelt. Aber ungeachtet des Zwistes fragt sich: erschöpft die pratyātmadharmatā oder das dharmadhātvātmakam jñānam alle Erkenntnisse und Lehren? Das erhobene badhicittam erreichte es, indem es den samisāra transzendiert und noch dazu jede frühere Stufen der Erkenntnisse und Lehren transzendiert. Aber in dem Augenblick, wo das erreichte mit dem Begriff des dharmadhātvātmakam jn̄ānam oder der pratyātmadharmatā ausgedrückt wird, eben in demselben Augenblick ist das bodhicittam dem Buddhismus eigen und immanent. Und der Absolutheitsanspruch wird von selbst verneint, denn der Buddhismus findet sich unter anderen Philosophien und Religionen, deren

\footnotetext{
(7)(8) Taisho Tripițakam, Bd. X, S. 102 A-B.
} 
jede eigenen Absolutheitsanspruch erhebt.

\section{Methodologische Relativierung}

Wenn aber jede Philosophie und Religion Absolutheitsanspruch erhebt, wird der Anspruch von selbst relativ, denn damit stehen viele Absolutheiten nebeneinander. Auf dem gegenwärtigen Stande der Universalgeschichte der Philosophie ist der Anspruch zu eilig. Wohl will jede absolut sein, aber das Wollen ist naiv und borniert und ihm fehlen strenge, unerbittliche Selbstkritik und wissenschaftliche Kritik der Anderen, weil die Grundvoraussetzungen der einen Philosophie verschieden von denen der Andern sind und so irgendeine Philosophie die Absolutheit für sich allein, ohne weiteres und in Ausschliessung der Anderen nicht beanspruchen kann. Und so wäre es methodologisch am besten, dass jede sich transzendiert und relativiert und vom Gesichtspuncte der Universalgeschichte der Philosophie sich und die Anderen in Bezug auf die Allgemeinheit (Universalität) genau prüft, die jede für sich beansprucht. Dabei hat die buddhistische Philosophie die Transzendenz-und Immanenznatur des bodhicittam als geeignete Methode zweckmässig in Gebrauch zu nehmen. Dies gilt aber nur von der buddhistischen Philosophie. Und jede Philosophie von der universalgeschichtlichen Grösse soll sich genau prüfen und aus sich heraus den Weg zur methodologischen Relativierung ausfindig machen. Sonst würde das gegenseitige Verständnis der Philosophien verloren gehen und würden nur bornierte Rechthaberei und gegenseitige Unterschätzung herrschen.

\section{Schlussbetrachtung}

Zur Erhellung dessen, was der Aufsatz vorschlägt; nehme man das 《Mahāyāna-śraddhotpāda Śāstra.》 Es stellt vier śraddhās auf: die der Wurzel, des Buddha, der Lehre des Buddha (dharma) und des Samgha. Gewöhnlich werden nur die drei letzteren aufgestellt, die sog. drei Juwelen. Wenn die vier aufgestellt werden, so stehen sie nicht nebeneinander. Die drei letzteren sind auf die erste eine gegründet, die die anderen möglich 
Transzendenz und Immanenz des Bodhicittam (Kumatara Kawada) (27) macht. Aus diesem Gedanken kann der Transzendenzgedanke sich sehr klar herausnehmen. Wenn einer nur die drei letzteren im Auge hat und nichts weiter weiss, so bleibt er den drei Juwelen nur immanent. Wer dagegen auch die Wurzel im Auge hat, der transzendiert die Juwelen und ist zugleich ihnen immanent, insofern er den buddhadharma verehrt. Und wer so tun kann, der kann die Wurzel mit anderen philosophischen rhizomaten vergleichend prüfen, weil er auf der einen Seite ganz frei von den drei Juwelen sein kann, auch wenn er auf der andern Seite ihnen immanent ist. Die Wurzel in diesem Śāstra ist die paurāṇasthitidharmatā im Lam̉kāvatāram, und die Lehre des buddha die pratyātmadharmatā. Wer diese letztere nicht transzendiern kann, der ist ein bornierter Gefangener des Buddhismus, der den Buddhismms nicht von innen und von aussen sehen und ihn genau kennen kann und die buddhistische Philosophie an den gebührenden Platz in der Universalgesschichte der Philosophie nicht setzen kann.

\section{FOREIGN INFLUENCE IN ANCIENT INDIA}

By R. A. Jairazbhoy

I The Indus Valley and Mesopotamia

II The Western Connexions of the Indo-Aryans

III Post-Vedic, Pre-Persian Influences in India

IV Indian Response to Persian Imperialism

$\mathrm{V}$ The impact of Greek Culture upon India

VI Roman Relations with India

VII Partho-Sassanian Exchanges with India

VIII India and the Muslims Prior to Permanent Conquest 195pp. Asia Publishing House, London. 1963. 